\title{
Raman Spectra in Irradiated Graphene: Line Broadening, Effects of Aging and Annealing
}

\author{
Issai Shlimak*, Moshe Kaveh \\ Jack and Pearl Resnick Institute of Advanced Technology, Department of Physics, Bar-Ilan University, Ramat-Gan, Israel \\ Email: ^ishlimak@gmail.com
}

How to cite this paper: Shlimak, I. and Kaveh, M. (2020) Raman Spectra in Irradiated Graphene: Line Broadening, Effects of Aging and Annealing. Graphene, 9, 13-28.

https://doi.org/10.4236/graphene.2020.92002

Received: March 8, 2020

Accepted: April 27, 2020

Published: April 30, 2020

Copyright $\odot 2020$ by author(s) and Scientific Research Publishing Inc. This work is licensed under the Creative Commons Attribution-NonCommercial International License (CC BY-NC 4.0). http://creativecommons.org/licenses/by-nc/4.0/ (c) (i) (8) Open Access

\begin{abstract}
The results of measurements of the Raman spectra in the same group of monolayer graphene samples, successively subjected to irradiation with different ions, prolonged aging, and annealing under different conditions, are considered. Changes in the position, width, and intensity of the Raman lines are analyzed in the study of the following problems: comparison of the results of irradiation with various ions, the influence of prolonged aging on the spectra of irradiated samples, the mechanism of broadening of Raman scattering lines caused by an increase in the density of radiation defects, the consequences of annealing of radiation damages in vacuum and in the atmosphere of the forming gas, the contribution of doping and lattice deformation to the shift of the position of the Raman lines after annealing. The results obtained made it possible to determine the level of stability of defects introduced by radiation, to reveal the possibility of restoring the damaged lattice using annealing. Since the results relate to graphene deposited on a widely used $\mathrm{SiO}_{2} / \mathrm{Si}$ substrate, they may be of interest when using ion irradiation to change the properties of graphene in appropriate devices.
\end{abstract}

\section{Keywords}

Graphene, Raman Spectra, Ion Irradiation, Annealing

\section{Introduction}

Modification of graphene properties is essential for the various electronic applications it offers [1] [2] [3] [4] [5]. For this purpose, ion irradiation is widely used (see, for example, [6] [7] [8] [9]) due to the possibility of controlling the ion energy and radiation dose with high accuracy. Irradiation of the pristine graphene samples leads to an increase in disorder due to the introduction of structural defects that affect its electrical and optical properties. 
Measurements of Raman scattering spectra (RS) are usually considered to be an effective tool for probing the structure of disordered graphene films and density of introduced defects [10] [11] [12] [13]. Typical RS spectra for disordered graphene consist of three main spectral lines. The G-line at $1600 \mathrm{~cm}^{-1}$ is common for different carbon-based materials, including carbon nanotubes, monoand multilayered graphene, and graphite. The $2 \mathrm{D}$ line at $2700 \mathrm{~cm}^{-1}$ belongs to the intervalley two-phonon mode, corresponds to the momentum conservation, and can be emitted from anywhere in the intact crystal structure, far from any defects. The "defective" D-line at $1350 \mathrm{~cm}^{-1}$ is associated with the one-phonon scattering, which is forbidden in an ideal graphene lattice due to conservation of momentum, but is possible in the vicinity of a lattice defect (edges and vacancies) due to additional elastic scattering by the defect. In addition, a few minor lines connected with different modes of phonons can be seen in disordered samples. The positions of all lines are given for $532 \mathrm{~nm}$ excitation laser. As a result, the intensity of the "defective" D-line is used as a measure of disorder in graphene layers (in the form of the ratio of the amplitudes of the D- and G-lines, $\alpha$ $\left.=I_{D} / I_{G}\right)$. Accordingly, the normalized intensity of the $2 \mathrm{D}$-line, $\beta=I_{2 D} / I_{G}$ can be used as a measure of the intact portion of the lattice.

Raman scattering in irradiated graphene has been studied for over ten years. Many works have been devoted to the study of RS in samples obtained by different methods and irradiated with different ions. Graphene films were supported by different substrates, which also influenced the irradiation result. As a result of a significant dependence of the results of ion irradiation on the method of preparation of the samples, the material of the substrate, the mass and energy of ions, the level of doping and the quality of initial samples, it is difficult to compare the results obtained in different works, sometimes they are even contradictory. A feature of the presented study is measurements on the same group of samples. Raman spectra were recorded in these samples sequentially after ion irradiation, prolonged exposure in air at room temperature, and annealing at various temperatures and conditions. Some results of different studies were partially reported earlier [14] [15] [16] [17], however, it was the presence of the same samples in each study that allowed, as the results were accumulated, to obtain a complete picture of the transformation of Raman spectra in graphene starting with irradiation and ending with annealing. This helped to determine the level of stability of defects introduced by radiation, to reveal the possibility of restoring the damaged lattice using annealing. Since the results relate to graphene deposited on a widely used $\mathrm{SiO}_{2} / \mathrm{Si}$ substrate, they may be of interest when using ion irradiation to change the properties of graphene in appropriate devices.

\section{Materials and Methods}

Two initial large-scale samples $(5 \times 5 \mathrm{~mm})$ of monolayer graphene were provided by Graphenea Co, Spain. The graphene film was obtained by chemical va- 
por deposition on a copper catalyst and transferred to a $300 \mathrm{~nm} \mathrm{SiO} / 2 \mathrm{Si}$ substrate using a wet transfer process. Graphene samples of such a large size were polycrystalline films with an average microcrystal size of about $10 \mu \mathrm{m}$. In Raman measurements, excitation was achieved by a laser beam of wavelength $\lambda=532$ $\mathrm{nm}$ and a power of less than $2 \mathrm{~mW}$ to avoid heating and destruction of the film. Reproducibility was verified by repeated measurements in the same point and in different samples from the same group.

Six groups of mini-samples $(0.2 \times 0.2 \mathrm{~mm})$ were prepared on the surface of the first large-scale sample using electron beam lithography (EBL). One group was not exposed to radiation, and five other groups were irradiated with different doses of $\mathrm{C}^{+}$ions with an energy of $35 \mathrm{keV}$. Such mini-samples were not made on the surface of the second large-scale sample. Instead, six small sections of the sample were irradiated with different doses of $\mathrm{Xe}^{+}$ions with the same energy of $35 \mathrm{keV}$, one region was not irradiated. Irradiation was performed on an HVEE-350 implanter. Thus, two series of samples of monolayer graphene with a common substrate irradiated with different ions were obtained.

The experimental results of measurements of the Raman spectra in these series of samples are presented and analyzed below. Theoretical support for the experiments consisted in the development of existing models as applied to the observed results. In particular, the results of computer simulation of the interaction of an impact ion with the graphene lattice were used [18], as well as the method for calculating the intensity [19] and the shape [20] of Raman lines in irradiated graphene.

In an ideal crystalline monolayer graphene, the shape of the one-phonon Raman line $I(\omega)$ could be described by a Lorentzian function [13]

$$
I(\omega) \propto \frac{1}{\left[\omega-\omega\left(q_{0}\right)\right]^{2}+[\Gamma / 2]^{2}}
$$

where $\omega$ is the frequency, $\omega\left(q_{0}\right)$ is the peak position of the RS line associated with emission of a phonon of the wave vector $q_{0}$ and $\Gamma$ is the full width at the half maximum (FWHM) which is reversely proportional to the phonon lifetime. In irradiated crystal, the size of correlation functions $L$ become finite due to the induced defects. At low disorder, the phonon wave packet can be described by a Gaussian function $\exp \left[-\left(q-q_{0}\right)^{2} L^{2} / 4\right]$ centered in $q_{0}$ and having a width proportional to $1 / L$. This leads to modification of $I(\omega)$ as [20]:

$$
I_{D}(\omega) \propto \int \mathrm{d} q \frac{\exp \left(\frac{-\left(q-q_{0}\right)^{2} L^{2}}{4}\right)}{\left[\omega-\omega_{\text {іто }}(q)\right]^{2}+\left[\Gamma_{0} / 2\right]^{2}}
$$

where $q$ is expressed in units of the reciprocal lattice constant, $q_{0}$ is measured from the K-point in the Brillouin zone, $\Gamma_{0}$ is the width of the unperturbed Raman line shape and $L$ is a phonon coherence length which should be of the order of the average distance between point defects $L_{d}$. Expression for the phonon dis- 
persion is taken in the form:

$$
\omega_{\text {іто }}(q)=\omega_{K}+A q
$$

By changing the integration variable, integral (2) can be performed in the Voigt distribution [21]. In spectroscopy, a Voigt profile results from convolution of two broadening mechanisms, one of which alone would produce a Lorentzian profile and the other would produce Gaussian profile. A rough approximation for the relation between the widths of the Voigt $f_{V}$, Gaussian $f_{G}$ and Lorentzian $f_{L}$ profiles is

$$
f_{V} \approx f_{L} / 2+\sqrt{\left(f_{L} / 2\right)^{2}+f_{G}^{2}},
$$

Using expressions for these profiles, we obtain [17]:

$$
f_{L}=\Gamma_{0}, \quad f_{G}=4(\ln 2)^{1 / 2} A / L
$$

This gives

$$
f_{V} \approx \frac{\Gamma_{0}}{2}+\sqrt{\frac{\Gamma_{0}^{2}}{4}+\frac{16(\ln 2) A^{2}}{L^{2}}}
$$

In the limit $f_{G} \gg f_{L}$ which corresponds to strong broadening $\left(\Delta \Gamma \gg \Gamma_{0}\right)$

$$
f_{V} \approx \frac{f_{L}}{2}+f_{G} \approx \frac{\Gamma_{0}}{2}+\frac{4 \sqrt{\ln 2} A}{L}
$$

so $\Delta \Gamma$ is proportional to $1 / L$.

In the opposite limit of weak broadening $f_{G} \ll f_{L}$, we obtain [17]:

$$
f_{V} \approx f_{L}+\frac{f_{G}^{2}}{f_{L}} \approx \Gamma_{0}+\frac{16(\ln 2) A^{2}}{\Gamma_{0} L^{2}}
$$

so $\Delta \Gamma \sim(1 / L)^{2}$. Since $L \approx L_{d}$, we obtain that $\Delta \Gamma \sim\left(1 / L_{d}\right)^{2} \sim N_{d}$, where $N_{d} \approx\left(1 / L_{d}\right)^{2}$ is the density of irradiation introduced defects. This analysis will be used in Section 3.3 to study the broadening of Raman lines with increasing radiation dose.

\section{Results}

In Figure 1, the RS spectra of samples irradiated with light $\left(\mathrm{C}^{+}\right)$and heavy $\left(\mathrm{Xe}^{+}\right)$ ions are shown. The changes in the intensity of the $\mathrm{D}$ - and the $2 \mathrm{D}$-lines are similar. In unirradiated samples, the intensity of the "defective" D-line is low or absent, which indicates a good quality of the initial monolayer graphene sample. With an increase of the irradiation dose $\Phi$, the D-line increases and then expands and decreases, while the 2D-line decreases monotonically and disappears. The only difference is in the dose range in which these changes are observed: upon irradiation with Xe ions, the values of $\Phi$ are an order of magnitude smaller than those under irradiation with $\mathrm{C}$ ions. Due to the equality of energies, this difference is associated with different ion masses.

To compare the results, it is necessary to plot the dependence of the normalized ratio $\alpha=I_{D} / I_{G}$ and $\beta=I_{2 D} / I_{G}$ not on the irradiation dose $\Phi$, but on the density of structural defects $N_{d}$, which is proportional to $\Phi, N_{d}=k \Phi$. The numerical 


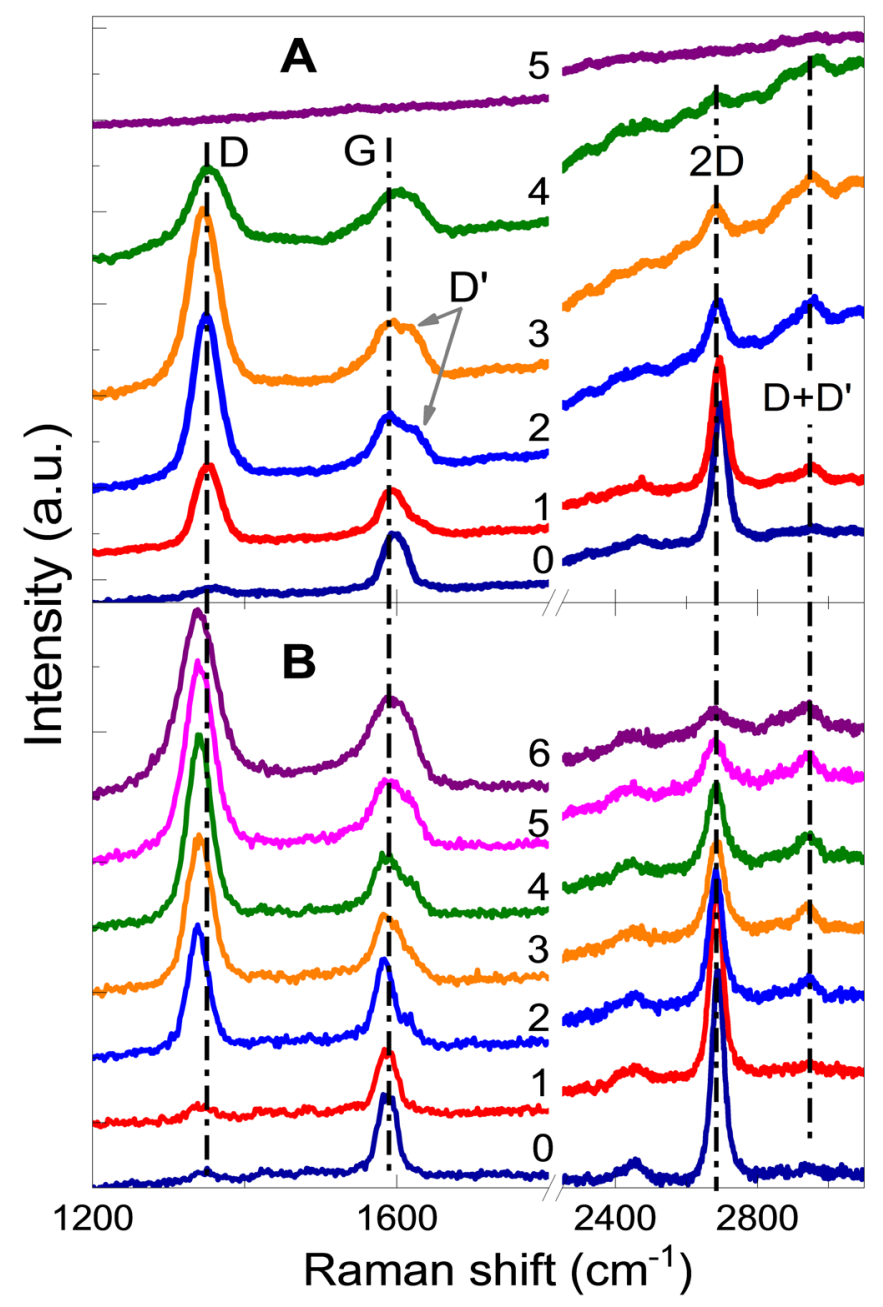

Figure 1. The Raman spectra for series of samples irradiated with $\mathrm{C}$ ions (A) and Xe ions (B). For the C-series, $\Phi$ in $10^{14} \mathrm{~cm}^{-2}$ : 0 -initial, $1-0$ (non-irradiated, but after EBL), $2-0.5,3-1.0,4-2.0,5-10$. For the Xe-series, $\Phi$ in $10^{13} \mathrm{~cm}^{-2}$ : 0 -initial, $1-0.15,2-0.3$, $3-0.5,4-1.0,5-2.0,6-4.0$.

coefficient $k$ depends on the energy $E$ and mass $M$ of the ion and reflects the average fraction of a carbon atom knocked out of the graphene lattice in one impact of the ion. Computer simulation of creation of different types of defects in monolayer graphene induced by ion irradiation has been performed in [18]. Based on these results, we calculated the dependences of $k$ on $M$ (in atomic mass units, amu) for various ion energy $E$ shown in Figure 2. For $E=35 \mathrm{keV}$, the value of $k$ for $\mathrm{C}$ ions $(M=12)$ is $k \approx 0.08$, while for Xe ions $(M=131), k \approx 0.8$, ten times larger, which explains the difference in $\Phi$. The dependences of $\alpha$ and $\beta$ on $N_{d}$ for both series of samples are shown in Figure 3. It is seen that on this scale the experimental data are indeed very close.

\subsection{Line Intensity}

Figure 3 shows that with the increase in $N_{d}$, the $I_{D}$ first increases up to a maximum value, and then decreases. Such non-monotonic behavior of $I_{D}$ is common 


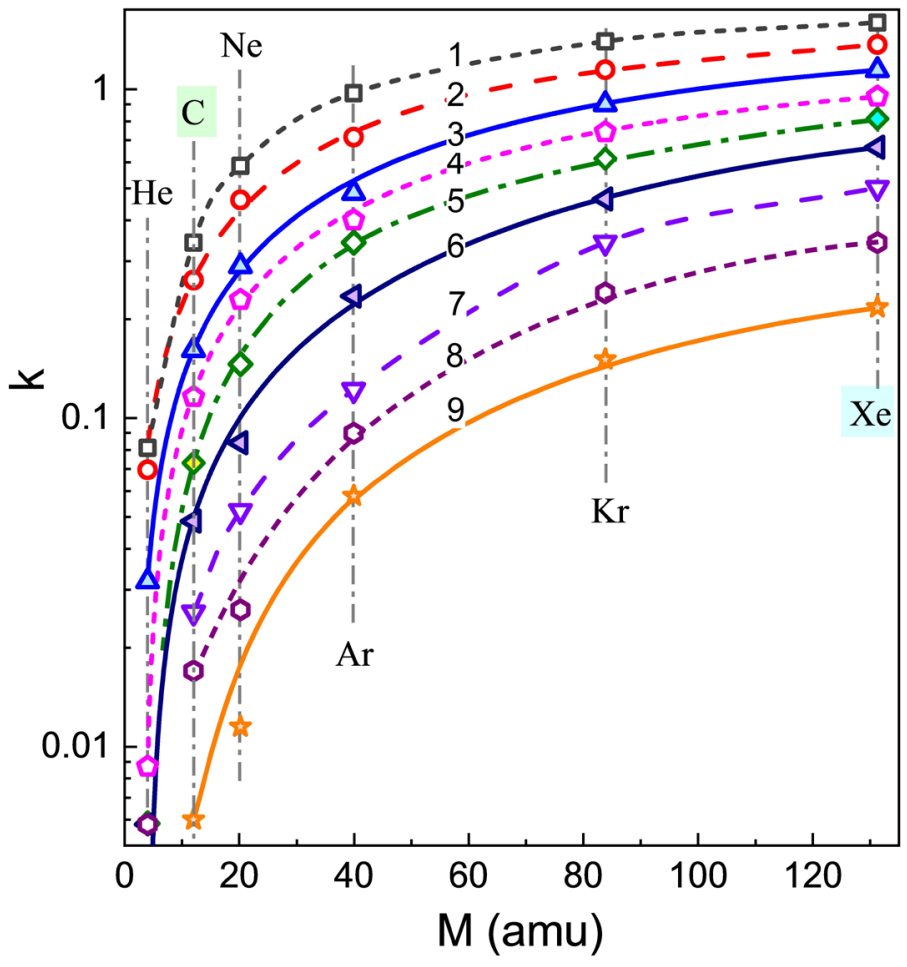

Figure 2. The plot of $k$ versus the ion mass $M$ (in amu) for different energies $E$ (in $\mathrm{keV}$ ): $1-0.4,2-1.1,3-5.0,4-14,5-35,6-100,7-270,8-730,9-1000$. Some ions are indicated with vertical dotted lines.

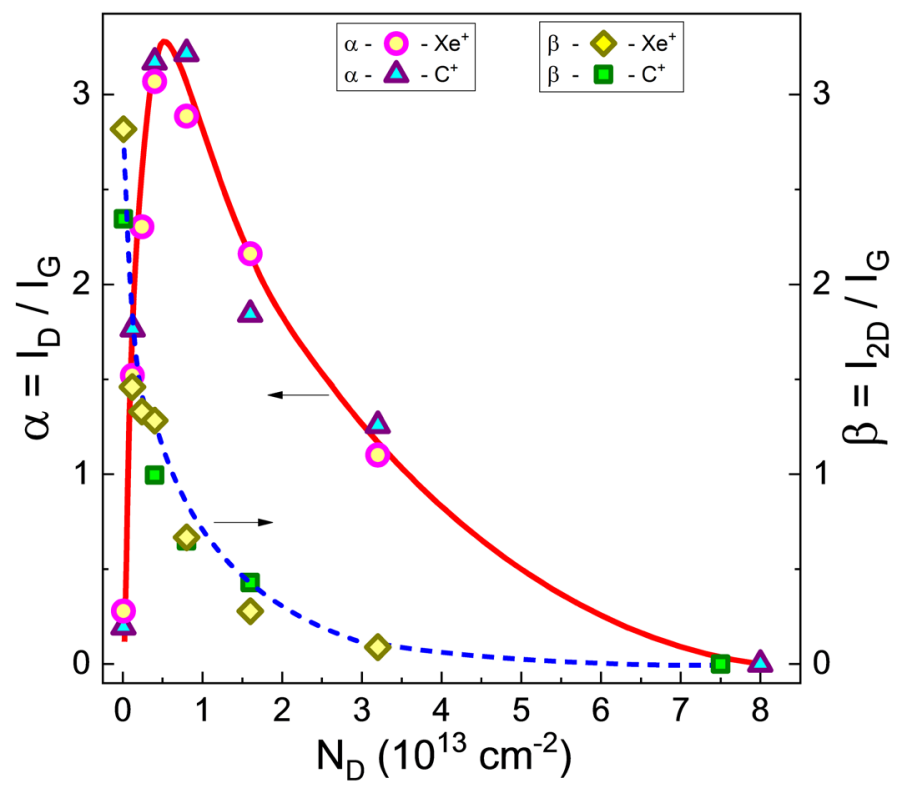

Figure 3. The normalized amplitude of the D-line $\alpha=I_{D} / I_{G}$ and the $2 \mathrm{D}$-line $\beta=I_{2 D} / I_{G}$ for samples irradiated with $\mathrm{C}$ ions and Xe ions as a function of $N_{d}$.

for many experimental observations [10] [11] [12] [13] and agrees with the theoretical model [19] based on the assumption that a single ion impact leads to the formation of a defect characterized by two length scales $r_{S}$ and $r_{A}\left(r_{A}>r_{S}\right)$, 
which are the radii of two circular areas surrounding the defect. The area $S$ within the shorter radius $r_{S}$ around the impact point is structurally disordered. In the $A$-area ("activated" area) between $r_{A}$ and $r_{S}$, the lattice structure is preserved, but the proximity to the impact point causes a breakdown of the selection rules and gives rise to the emission of the "defective" D-peak. The 2D-peak, which is characteristic for a perfect graphene lattice, can be generated mostly if the electron-hole excitation occurs outside the $A$-area. Increasing the irradiation dose obviously results in an increase of the D-line intensity and a decrease of the 2D-line intensity. Increasing of $N_{d}$ leads also to a decrease of the average distance between defects $L_{d} \approx\left(N_{\mathrm{d}}\right)^{-1 / 2}$, and when $L_{d}$ becomes shorter than $r_{A}$, the $A$-areas begin to overlap with each other and with $S$-areas. As a result, $\alpha=I_{D} / I_{G}$ passes through a maximum and then decreases.

The equation for $\alpha=I_{D} / I_{G}$ vs. $L_{d}$ was obtained in [19] and has the following form after minor correction in [14]:

$$
\alpha=C_{A} \mathrm{e}^{-\pi r_{S}^{2} / L_{d}^{2}}\left[1-\mathrm{e}^{-\pi\left(r_{A}^{2}-r_{S}^{2}\right) / L_{d}^{2}}\right]+C_{S}\left[1-\mathrm{e}^{-\pi r_{S}^{2} / L_{d}^{2}}\right]
$$

where $C_{A}$ is the maximal possible value of $\alpha$ in graphene which could be attained for the situation in which no damage occurred to the hexagonal network of carbon atoms, and $C_{S}$ is the $I_{D} / I_{G}$ value in the highly disordered limit.

The dependencies of $\alpha$ on $L_{d}$ for both series of samples are presented in Figure 4, together with the best fit of Equation (8) shown as solid lines 1 and 2. It can be seen that they are slightly different, which indicates a difference in the size of defects produced by different ions. The best fit allows you to determine the adjustable parameters in Equation (8): for the C-series we have $C_{A}=5.4, C_{S}=0, r_{S}$

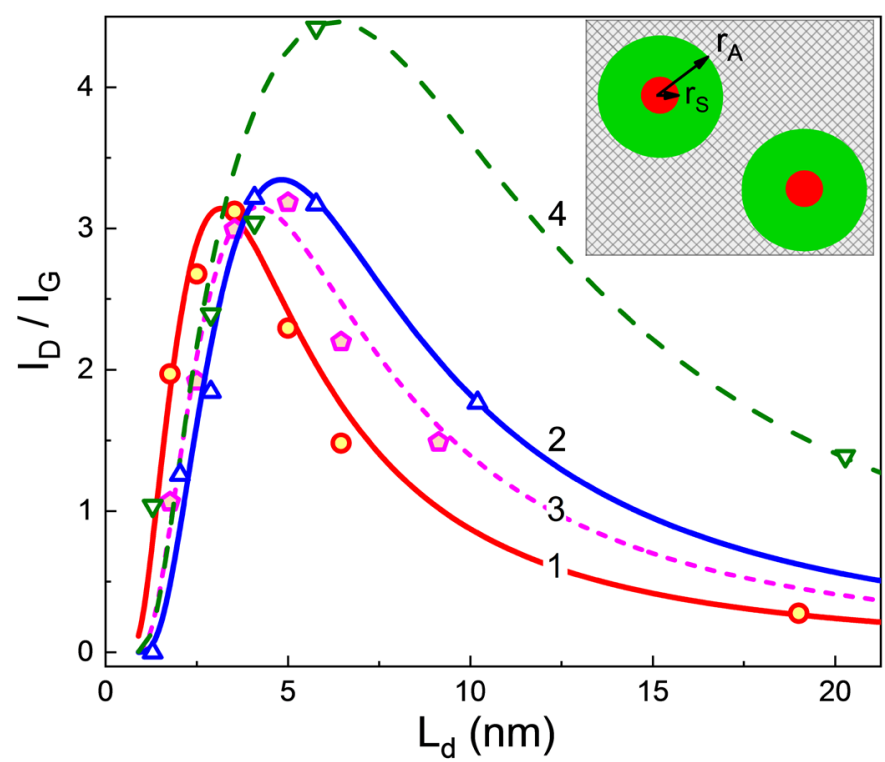

Figure 4. Dependence of $\alpha=I_{D} / I_{G}$ on the average distance between defects $L_{d}$ for samples irradiated with $\mathrm{Xe}^{+}$and $\mathrm{C}^{+}$ions just after irradiation $(1,2)$ and after long-term aging $(3$, 4). Solid and dashed lines represent the fit to Equation (1) with the parameters specified in the text. The inset shows schematically two defect radii. 
$=1.55 \mathrm{~nm}, r_{A}=4.1 \mathrm{~nm}$, and for the Xe-series $C_{A}=4.9, C_{S}=0, r_{S}=1.0 \mathrm{~nm}, r_{A}=$ $2.8 \mathrm{~nm}$. Comparison shows that irradiation with $\mathrm{C}$ ions is characterized by higher $r_{S}$ and $r_{A}$ values than irradiation with Xe ions. This may be due to the effect of backscattering when the $\mathrm{SiO}_{2}$ substrate is bombarded with carbon ions that are lighter than the substrate atoms, as opposed to irradiation with heavier Xe ions.

\subsection{Sample Aging}

The Raman spectra in our samples were measured after long-term storage for about a year at room temperature in air. On the one hand, the surrounding atmosphere is not fully reproduced in different places, but on the other hand, most devices are stored at room temperature in air, so this study may be of interest to specialists in graphene-based devices.

Prolonged aging leads to noticeable but not fundamental changes in RS, which indicates that the ion irradiation introduced defects are quite stable. Figure 5 shows the spectra "just after irradiation" and "after aging" for two samples from Xe-series. It can be seen that while the $2 \mathrm{D}$-line is always weaker in the "aged" samples, the D-line behaves in the opposite way: the line intensity increases for weakly irradiated samples, but decreases for strongly irradiated ones. Different behavior of the D-line becomes clear when we plot dependences $\alpha\left(L_{d}\right)$ for "aged" samples (dashed lines 3 and 4 in Figure 4). Fitting to theoretical expression Equation (8) gives new values for the size of defects (in parentheses - the previous values immediately after irradiation): for C-series $r_{S}=1.35(1.55) \mathrm{nm}, r_{A}=6.4$ (4.1) $\mathrm{nm}$, for Xe-series $r_{S}=1.3(1.0) \mathrm{nm}, r_{A}=3.7(2.8) \mathrm{nm}$. We see that the long-term aging leads to an increase in $r_{A}$. The increase in the square of $A$-area

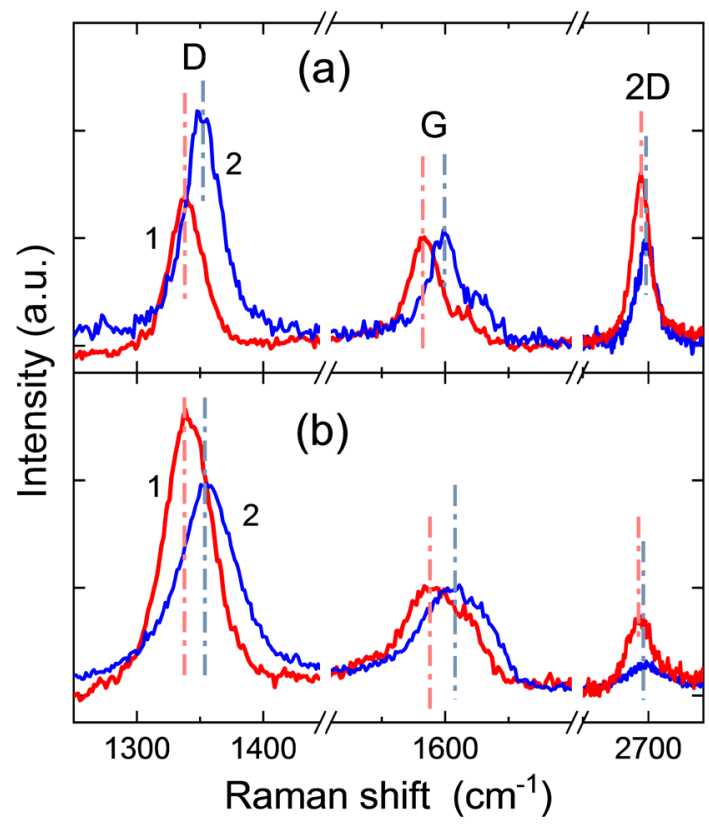

Figure 5. Comparison of the normalized RS spectra in "just irradiated" (1) and "aged" (2) samples from the Xe-series with different density of defects. $N_{d}$, in $10^{13} \mathrm{~cm}^{-2}$ : (a) -0.3 , (b) -2.0 . Intensity of G-line is equal to one. 
could be caused, probably, by the capture of molecules from the ambient atmosphere This explains the different behavior of the D-line for weakly and strongly irradiated samples shown in Figure 5. For weakly irradiated samples, the $\mathrm{D}$-line increases because of the increase in the square of $A$-areas. However, the overlap of increased $A$-areas begins earlier, at a lower density of defects, which explains the decrease in the D-line in strongly irradiated samples. Accordingly, the intact area is reduced, resulting in a smaller $2 \mathrm{D}$ line.

One can see also from Figure 5 that the peak positions in the "aged" samples shift to higher frequencies ("blue shift"). A possible origin for the blue shift is the influence of doping [22]. The doping scenario agrees with the assumption that the increase of $r_{\mathrm{A}}$ in "aged" samples is caused by the capture of molecules from the ambient atmosphere.

\subsection{Line Broadening}

In the RS process, an absorbed photon generates an electron-hole pair and the excited electron with momentum $\boldsymbol{k}$ is further scattered by phonons with momentum $\boldsymbol{q}$ following by recombination with hole and emission of a photon which is red-shifted by the amount of energy given to the phonon. Due to momentum conservation requirements, the first-order RS process generates only phonons with wave vector $\boldsymbol{q}=0$ at the $\Gamma$ point in the center of the first Brillouin zone. This band in RS spectra is known as G-band $\left(\sim 1600 \mathrm{~cm}^{-1}\right)$ and exists for all $s p^{2}$ carbon system, including amorphous carbon, carbon nanotubes and graphite.

In disordered graphene, when the lattice periodicity is broken near defects, the momentum conservation requirement is also broken. At first sight, in this case all phonons in the Brillouin zone are allowed for the RS, but the energy conservation requirement allocates in the resonant electron-phonon scattering some selected phonon modes [23] which form the "defective" D-band ( 1350 $\mathrm{cm}^{-1}$ ) with emission of in-plane transverse optic phonon (iTO) accompanied by an elastic scattering on defect. The intense $2 \mathrm{D}$-line $\left(\sim 2700 \mathrm{~cm}^{-1}\right)$ observed in purely crystalline pristine graphene corresponds to second-order RS process with excitation of two iTO-phonons with opposite momenta.

We are aware of only a few works in which the change in the line width $\Gamma$ (full width at half maximum, FWHM) in irradiated graphene is studied [20] [24] [25]. In Ref. [20], the increase of $\Gamma$ is analyzed as a function of $1 / L_{d} \approx\left(N_{d}\right)^{1 / 2}$. In Ref. [24], it is shown that $\Gamma$ increases sublinearly with the increase in $N_{d}$, while in Ref. [25], this dependence is shown as superlinear at a high level of disorder. Such an uncertainty along with small number of works in this field motivated our study.

Our measurements presented in Figure 6 show that broadening of all RS lines is linear with increasing $N_{d .} \Gamma=\Gamma_{0}+m N_{d}$, where $\Gamma_{0}$ is the FWHM in the pristine sample and the coefficient $m$ (in $\mathrm{cm}$ ) characterizes the broadening rate. The linear dependence $\Delta \Gamma=\Gamma-\Gamma_{0}$ on $N_{d}$ is correctly described by theoretical expression, 


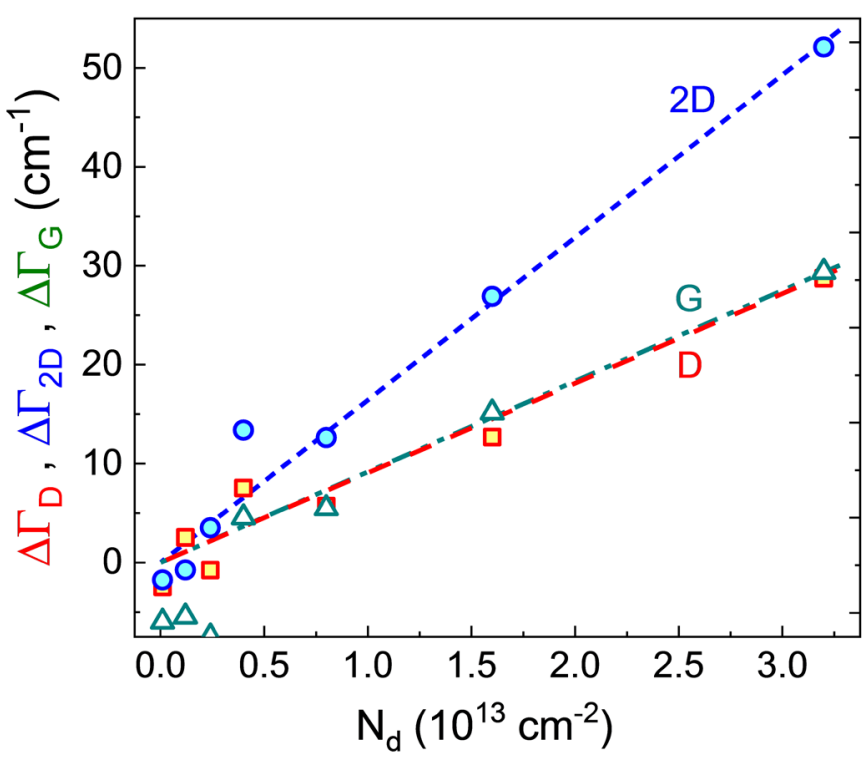

Figure 6. Dependences of $\Gamma_{D}, \Gamma_{G}$ and $\Gamma_{2 D}$ on the density of defects $N_{d}$ for the $\mathrm{Xe}^{+}$-series of samples. Dashed lines show the linear approximation.

Equation (7) can be explained by the fact that in our case, the broadening associated with Gaussian profile $f_{G}$ is not larger than the initial line width $\Gamma_{0}$ associated with Lorentzian profile $f_{L}$.

For single-phonon G- and D-lines, the broadening rates are equal $\left(m_{D, G}=0.9\right.$ $\left.\times 10^{-12} \mathrm{~cm}\right)$, while for $2 \mathrm{D}$-line, $m$ is almost two times higher $\left(m_{2 D}=1.7 \times 10^{-12}\right.$ $\mathrm{cm})$. This fact, together with the doubled energy of the peak position $\left(\omega_{2 D}=\right.$ $\left.2 \omega_{D}\right)$, reflects the widely used description of the $2 \mathrm{D}$-line as a second-order $\mathrm{D}$-feature. For the first glance, the width of the 2D-line caused by emission of two identical iTO phonons has to be double the width of the D-line. However, $\Gamma_{D}$ is less than one half of $\Gamma_{2 D}$, which can be seen in Figure 7 where both D- and 2D-lines are plotted together, but the upper scale for 2D-line is halved. The peak positions of D-line $\omega_{D}$ and the half of peak positions of 2D-line $\left(\omega_{2 D} / 2\right)$ coincide which confirms the elastic character of scattering by structural defects, so the energy of the emitted light quanta is changed only due to generation of one or two identical iTO phonons. However, it is also seen that the width of D-line is larger than one half of 2D-line. This difference could be attributed to the peculiarity of the D-band process which, by contrast with 2D-band, involves also an elastic scattering of electrons by a point defect which decreases the lifetime of emitted phonon and correspondingly leads to an additional broadening of the D-line.

\subsection{Annealing}

Ion irradiation as a method to introduce disorder is also interesting due to the possible reversibility of the radiation damage by annealing. There is extensive literature available on annealing of mono- and multi-layered graphene films. For the most part, however, these papers report the use of annealing in pristine, 


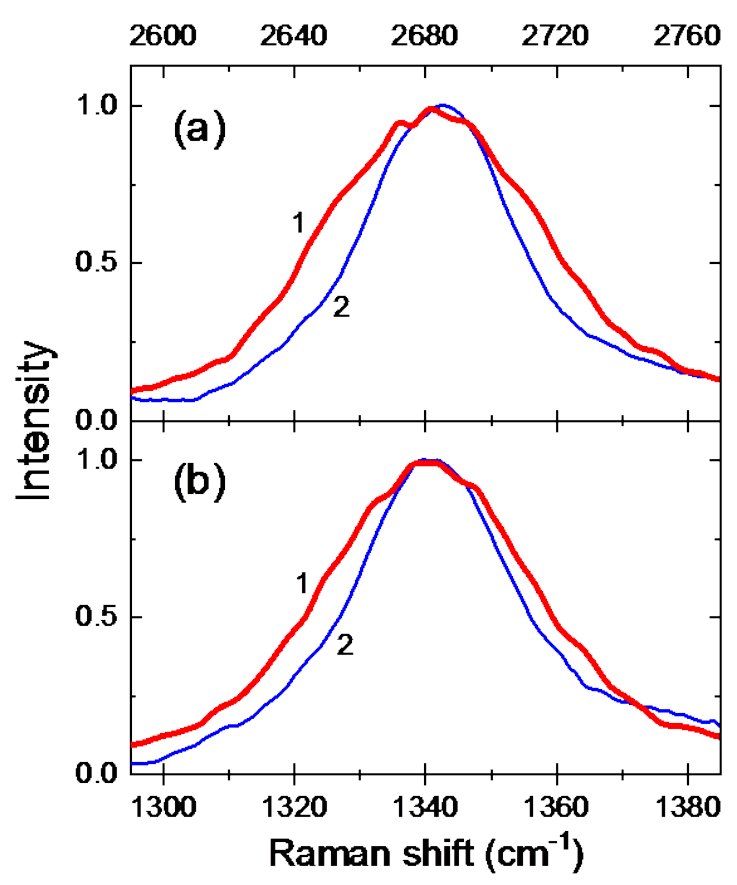

Figure 7. The normalized D-line (1, lower scale), and the 2D-line (2, upper doubly suppressed scale), for two samples from the Xe series. $N_{d}\left(10^{13} \mathrm{~cm}^{-2}\right)$ : (a) -0.24 , (b) -0.4 .

non-irradiated graphene as a procedure for unintentional doping and the removal of polymer residues which remain after either wet graphene transfer to the substrate or photolithography used in the device processing [26] [27] [28] [29]. In a few works, annealing was applied to samples irradiated either with ions [30] [31], or UV light [32].

Our irradiated samples from Xe- and C-series were annealed after long-term aging described in subsection 3.2. Annealing the Xe-series samples was performed in high vacuum $\left(4 \times 10^{-6}\right.$ Torr $)$, while samples from the C-series were annealed in the mixed forming gas: $95 \% \mathrm{Ar}+5 \% \mathrm{H}_{2}$. In this case, the tube was pumped and purged to a pressure about 100 Torr before turning on the gas flow $(800 \mathrm{sccm})$. Samples were heated at a rate of $15^{\circ} \mathrm{C} / \mathrm{min}$ to different annealing temperature $T_{a}$ and then annealed for 1 hour. Cooling of the samples was performed by shutting off the heater and allowing the samples to cool naturally. The Raman spectra measured after each annealing at $T_{a}=500^{\circ} \mathrm{C}, 800^{\circ} \mathrm{C}$ and $1000^{\circ} \mathrm{C}$ showed strong influence of the annealing conditions on the intensity and the peak position of RS lines [16]. In Figure 8, the dependencies $\alpha$ and $\beta$ on $N_{d}$ for Xe-series samples are shown before and after annealing at $1000^{\circ} \mathrm{C}$. One can see that the D-line decreases, especially in weakly irradiated samples, while the 2D-line increases and even appears in highly irradiated samples. This means that annealing in vacuum leads to partial restoration of the damaged structure. Figure 9 shows that annealing in the forming gas is not as effective.

Forming gas and vacuum annealing result in a blue shift in the position of all three spectral lines, D, G and 2D. This effect can have two reasons: 1) hole doping caused by the increased ability to absorb oxygen and water molecules after 


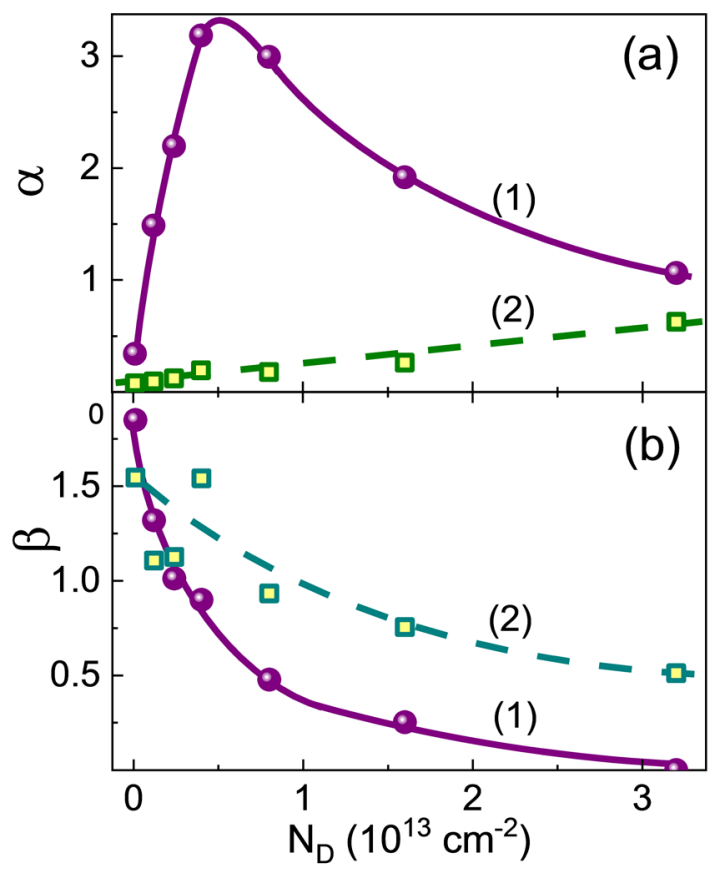

Figure 8. $\alpha=I_{D} / I_{G}$ (a) and $\beta=I_{2 D} / I_{G}$ (b) before (1) and after annealing at $1000^{\circ} \mathrm{C}(2)$ in vacuum for Xe-series samples. The lines are the guide to the eyes.

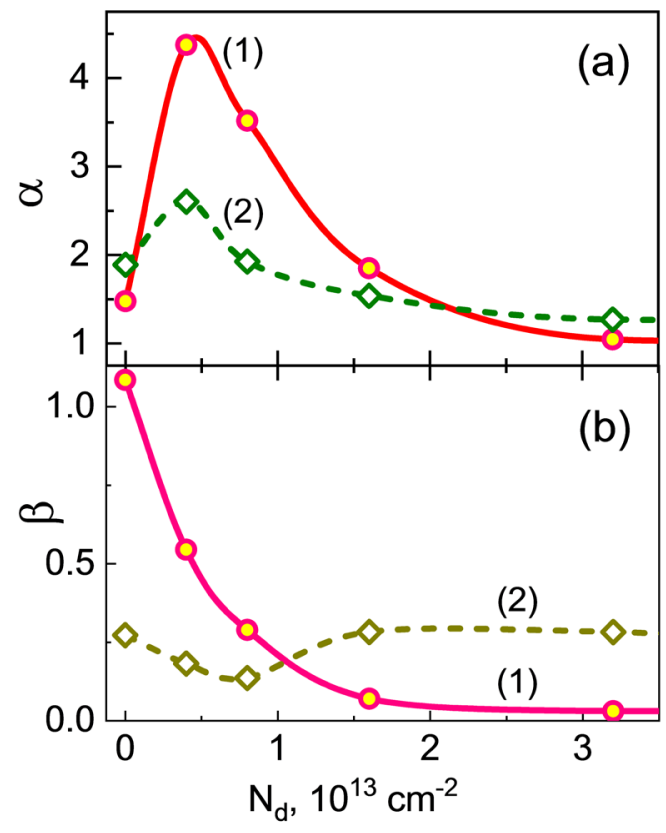

Figure 9. $\alpha=I_{D} / I_{G}$ (a) and $\beta=I_{2 D} / I_{G}$ (b) before (1) and after annealing at $1000^{\circ} \mathrm{C}(2)$ in forming gas for $\mathrm{C}$-series samples. The lines are the guide to the eyes.

annealing [33]; 2) compressive stress caused by the difference in thermal expansion of graphene and the substrate, sliding of the graphene film over the substrate during heating, followed by pinning of the annealed film upon cooling to room temperature [31]. It was shown in Ref. [34] that these two reasons can be distinguished when one plots the frequency (position) of the 2D-peak versus the 


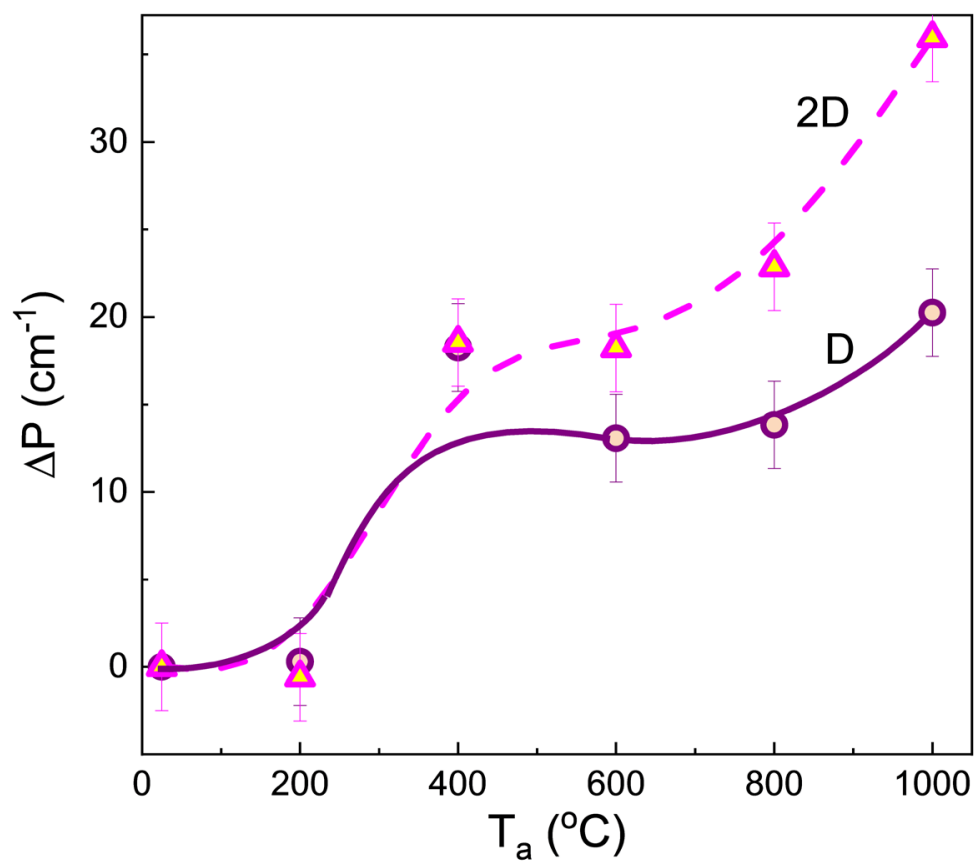

Figure 10. Shift of the average position of the 2D- and D-peaks for samples annealed in forming gas at different $T_{a}$. Error bars show spreading of the peak position for samples with different $N_{d}$.

position of the G-peak. When the doping effect dominates, the slope of this dependence is equal to 0.7 , while the domination of strain increases this slope to 2.2. The plotting of this dependence for our samples [16] showed for both series a straight line with a slope of about 1.0 , which means that the strain makes a significant contribution to the shift. There is another argument to justify the contribution of the strain. Lattice deformation leads to a change in the phonon energy. Therefore, the shift of the $2 \mathrm{D}$-line connected with emission of two equal phonons has to be double the shift of the $\mathrm{D}$-line. Figure 10 shows the shift $\Delta P$ of the position of both Raman lines after annealing at different $T_{a}$. One can see that below $T_{a}=500^{\circ} \mathrm{C}$ both shifts are equal, which indicates that at the low-temperature annealing, the shift is mainly caused by doping. With a further increase in $T_{a}$, the shift of the 2D-line rapidly increases, and the final shift is indeed about twice as large as the shift of the D-line. This indicates that at high annealing temperatures, the main cause of the shift is the residual stress of the graphene lattice.

\section{Conclusions}

Measurements on the same group of samples made it possible to obtain a complete picture of the transformation of the Raman spectra in monolayer graphene, beginning with irradiation and ending with annealing. The main results are as follows:

Changes in the line intensity caused by irradiation with different ions can be scaled into one common dependence.

Radiation-induced defects are relatively stable: only a slight increase in the 
size of the damaged area around each structural defect was observed after one year of aging.

The width of Raman lines increases linearly with increasing the density of irradiation-induced defects, which is explained by the developed theoretical model.

High-temperature annealing in vacuum leads to partial restoration of the damaged lattice, in contrast with annealing in forming gas.

After high-temperature annealing, the deformation of the graphene lattice is retained which leads to a change in the energy of in-plane transverse optical phonon (iTO).

Note that the results obtained refer to graphene films deposited on a widely used $\mathrm{SiO}_{2} / \mathrm{Si}$ substrate and, therefore, may be of interest when using ion irradiation to change the properties of deposited graphene and corresponding devices. A similar cycle is planned for studying samples of suspended or "free standing" graphene.

\section{Acknowledgements}

We are grateful to A. Butenko for helping with the drawings, E. Zion, E. Kogan, Yu. Kaganovsky, V. Richter, L. Wolfson, A. Sharoni, who took part in the measurements at different stages of the work.

\section{Conflicts of Interest}

The authors declare no conflicts of interest regarding the publication of this paper.

\section{References}

[1] Novoselov, K.S., Geim, A.K., Morozov, S.V., Jiang, D., Zhang, Y., Dubonos, S.V., Grigorieva, I.V. and Firsov, A.A. (2004) Electric Field Effect in Atomically Thin Carbon Films. Science, 306, 666-669.

[2] Kim, K., Choi, J.Y., Kim, T., Cho, S.H. and Chung, H.J. (2011) A Role for Graphene in Silicon-Based Semiconductor Devices. Nature, 479, 338-344. https://doi.org/10.1038/nature10680

[3] Schedin, F., Geim, A.K., Morozov, S.V., Hill, E.W., Blake, P., Katsnelson, M.I. and Novoselov, K.S. (2007) Detection of Individual Gas Molecules Adsorbed on Graphene. Nature Materials, 6, 652-655. https://doi.org/10.1038/nmat1967

[4] Yin, Z., Sun, S., Salim, T., Wu, S., Huang, X., He, Q., Lam, Y.M. and Zhang, H. (2010) Organic Photovoltaic Devices Using Highly Flexible Reduced Graphene Oxide Films as Transparent Electrodes. ACS Nano, 4, 5263-5268. https://doi.org/10.1021/nn1015874

[5] Schneider, G.F., Kowalczyk, S.W., Calado, V.E., Pandraud, G., Zandbergen, H.W., Vandersypen, L.M.K. and Dekker, C. (2010) DNA Translocation through Graphene Nanopores. Nano Letters, 10, 3163-3167. https://doi.org/10.1021/nl102069z

[6] Wang, Q., Mao, W., Ge, D., Zhang, Y., Shao, Y. and Ren, N. (2013) Effects of Ga Ion-Beam Irradiation on Monolayer Grapheme. Applied Physics Letters, 103, Article ID: 073501. https://doi.org/10.1063/1.4818458

[7] Guo, B., Liu, Q., Chen, E., Zhu, H., Fang, L. and Gong, J.R. (2010) Controllable 
N-Doping of Graphene. Nano Letters, 10, 4975-4980. https://doi.org/10.1021/nl103079j

[8] Buchowicz, G., Stone, P.R., Robinson, J.T., Cress, C.D., Beeman, J.W. and Dubon, O.D. (2011) Correlation between Structure and Electrical Transport in Ion-Irradiated Graphene Grown on Cu Foils. Applied Physics Letters, 98, Article ID: 032102. https://doi.org/10.1063/1.3536529

[9] Nanda, G., Goswami, S., Watanabe, K., Taniguchi, T. and Alkemade, P.F.A. (2015) Defect Control and n-Doping of Encapsulated Graphene by Helium-Ion-Beam Irradiation. Nano Letters, 15, 4006-4012. https://doi.org/10.1021/acs.nanolett.5b00939

[10] Ferrari, A.C. and Basko, D.M. (2013) Raman Spectroscopy as a Versatile Tool for Studying the Properties of Grapheme. Nature Nanotechnology, 8, 235-246.

https://doi.org/10.1038/nnano.2013.46

[11] Jorio, A., Lucchese, M.M., Stavale, F., Ferreira, E.H.M., Moutinho, M.V.O., Capaz, R.B. and Achete, C.A. (2010) Raman Study of Ion-Induced Defects in N-Layer Graphene. Journal of Physics: Condensed Matter, 22, Article ID: 334204. https://doi.org/10.1088/0953-8984/22/33/334204

[12] Dresselhaus, M.S., Jorio, A., Filho, A.G.S. and Saito, R. (2010) Defect Characterization in Graphene and Carbon Nanotubes Using Raman Spectroscopy. Philosophical Transactions of the Royal Society A, 368, 5355. https://doi.org/10.1098/rsta.2010.0213

[13] Saito, R., Hofmann, M., Dresselhaus, G., Jorio, A. and Dresselhaus, M.S. (2011) Raman Spectroscopy of Graphene and Carbon Nanotubes. Advances in Physics, 60, 413-550. https://doi.org/10.1080/00018732.2011.582251

[14] Shlimak, I., Haran, A., Zion, E., Havdala, T., Kaganovskii, Y., Butenko, A.V., Wolfson, L., Richter, V., Naveh, D., Sharoni, A., Kogan, E. and Kaveh, M. (2015) Raman Scattering and Electrical Resistance of Highly Disordered Grapheme. Physical Review B, 91, Article ID: 045414. https://doi.org/10.1103/PhysRevB.91.045414

[15] Butenko, A., Zion, E., Kaganovskii, Y., Wolfson, L., Richter, V., Sharoni, A., Kogan, E., Kaveh, M. and Shlimak, I. (2016) Influence of Ageing on Raman Spectra and the Conductivity of Monolayer Graphene Samples Irradiated by Heavy and Light Ions. Journal of Applied Physics, 120, Article ID: 044306.

https://doi.org/10.1063/1.4959880

[16] Zion, E., Butenko, A., Kaganovskii, Y., Wolfson, L., Richter, V., Sharoni, A., Kogan, E., Kaveh, M. and Shlimak, I. (2017) Effect of Annealing on Raman Spectra of Monolayer Graphene Samples Gradually Disordered by Ion Irradiation. Journal of Applied Physics, 121, Article ID: 114301. https://doi.org/10.1063/1.4978312

[17] Shlimak, I., Butenko, A., Kogan, E. and Kaveh, M. (2019) Irradiation-Induced Broadening of the Raman Spectra in Monolayer Grapheme. Journal of Applied Physics, 126, Article ID: 194302. https://doi.org/10.1063/1.5111354

[18] Lehtinen, O., Kotakoski, J., Krasheninnikov, A.V., Tolvanen, A., Nordlund, K. and Keinonen, J. (2010) Effects of Ion Bombardment on a Two-Dimensional Target: Atomistic Simulations of Graphene Irradiation. Physical Review B, 81, Article ID: 153401. https://doi.org/10.1103/PhysRevB.81.153401

[19] Lucchese, M.M., Stavale, F., Martins Ferreira, E.H., Vilani, C., Moutinho, M.V.O., Rodrigo, B., Capaz, R.B., Achete, C.A. and Jorio, A. (2010) Quantifying Ion-Induced Defects and Raman Relaxation Length in Grapheme. Carbon, 48, 1592-1597. https://doi.org/10.1016/j.carbon.2009.12.057

[20] Martins Ferreira, E.H., Moutinho, M.V.O., Stavale, F., Lucchese, M.M., Capaz, R.B., 
Achete, C.A. and Jorio, A. (2010) Evolution of the Raman Spectra from Single-, Few-, and Many-Layer Graphene with Increasing Disorder. Physical Review B, 82, Article ID: 125429. https://doi.org/10.1103/PhysRevB.82.125429

[21] Temme, N.M. (2010) Voigt Function. In: Olver, W.J., Frank, L., Daniel, M., Boisvert, R.F. and Clark, C.W., Eds., NIST Handbook of Mathematical Functions, Cambridge University Press, Cambridge, 159-172.

[22] Das, A., Pisana, S., Chakraborty, B., Piscanes, S., Saha, S.K., Waghmare, U.V., Novoselov, K.S., Krishnamurthy, H.R., Geim, A.K., Ferrari, A.C. and Sood, A.K. (2008) Monitoring Dopants by Raman Scattering in an Electrochemically Top-Gated Graphene Transistor. Nature Nanotechnology, 3, 210-215.

https://doi.org/10.1038/nnano.2008.67

[23] Malard, M.L., Pimenta, M.A., Dresselhaus, G. and Dresselhaus, M.S. (2009) Raman Spectroscopy in Grapheme. Physics Reports, 473, 51-87.

https://doi.org/10.1016/j.physrep.2009.02.003

[24] Zhou, Y.-B., Liao, Z.-M., Wang, Y.-F., Duesberg, G.S., Xu, J., Fu, Q., Wu, X.-S. and Yu, D.-P. (2010) Ion Irradiation Induced Structural and Electrical Transition in Graphene. The Journal of Chemical Physics, 133, Article ID: 234703. https://doi.org/10.1063/1.3518979

[25] Childres, I., Jauregui, L.A., Tian, J. and Chen, Y.P. (2011) Effect of Oxygen Plasma Etching on Graphene Studied Using Raman Spectroscopy and Electronic Transport Measurements. New Journal of Physics, 13, Article ID: 025008. https://doi.org/10.1088/1367-2630/13/2/025008

[26] Kumar, K., Kim, Y.S. and Yang, E.H. (2013) The Influence of Thermal Annealing to Remove Polymeric Residue on the Electronic Doping and Morphological Characteristics of Grapheme. Carbon, 65, 35-45. https://doi.org/10.1016/j.carbon.2013.07.088

[27] Wang, X.S., et al. (2013) Thermal Annealing of Exfoliated Graphene. Journal of Nanomaterials, 2013, Article ID: 101765. https://doi.org/10.1155/2013/101765

[28] Lin, Y.C., Lu, C.C., Yeh, C.H., Jin, C., Suenaga, K. and Chiu, P.W. (2012) Graphene Annealing: How Clean Can It Be? Nano Letters, 12, 414-419. https://doi.org/10.1021/nl203733r

[29] Sojoudi, H., Baltazar, J., Henderson, C. and Graham, S. (2012) Impact of Post-Growth Thermal Annealing and Environmental Exposure on the Unintentional Doping of CVD Graphene Films. Journal of Vacuum Science \& Technology B, 30, Article ID: 041213. https://doi.org/10.1116/1.4731472

[30] Edera, M. and Zaitsev, A.M. (2015) Ion Irradiation of Nanocrystalline Graphene on Quartz and Sapphire.

[31] Jia, K., Su, Y., Chen, Y., Luo, J., Yang, J., Lv, P., Zhang, Z., Zhu, H., Zhao, C. and Ye, T. (2015) Effects of Defects and Thermal Treatment on the Properties of Graphene. Vacuum, 116, 90-95. https://doi.org/10.1016/j.vacuum.2015.03.003

[32] Imamura, G. and Saiki, K. (2015) Modification of Graphene/ $/ \mathrm{SiO}_{2}$ Interface by UV-Irradiation: Effect on Electrical Characteristics. ACS Applied Materials \& Interfaces, 7, 2439-2443. https://doi.org/10.1021/am5071464

[33] Ni, Z.H., Wang, H.M., Luo, Z.Q., Wang, Y.Y., Yu, T., Wu, Y.H. and Shen, Z.X (2010) The Effect of Vacuum Annealing on Grapheme. Journal of Raman Spectroscopy, 41, 479-483. https://doi.org/10.1002/jrs.2485

[34] Lee, J.E., Ahn, G., Shim, J., Lee, Y.S. and Ryu, S. (2012) Optical Separation of Mechanical Strain from Charge Doping in Grapheme. Nature Communication, 3, Article No. 1024. https://doi.org/10.1038/ncomms2022 